theorotical basis for modular design, the theory of microwave techniques for moisture measurement, and the theory of sound transmission through lightweight partitions. Building practice is concerned with external contracts, technical enquiries, co-ordination of work on British Standards and Codes of Practice, building legislation, otc. The tropical building section is concerned with special problems of housing, building and planning in tropical and subtropical countries. Apart from these instructive demonstrations, facilities were available for providing technical information, in the form of leaflets giving background information and details of the researches involved in many of the exhibits; the technical enquiry service was in operation, dealing with questions on the technical aspects of building construction; the Station library was open to visitors and various official publications of the Station were displayed. In this latter connexion an important publication has since appeared entitled Developments in Roofing (Building Researeh Station Digest 51 (Second Series), October 1964; pp. 6, 4d.). This Digest discusses the proporties, construction and laying of roof decks and the wcatherproof coverings applied to obtain satisfactory performance. It also deals briefly with the properties of some new roofing materials. This is an excellent and up-to-dato summary of both conventional and unconventional roofing practices (excluding tiling) and a copy should be availablo to architects and all others concerned with modern building construction.

\title{
STRUCTURE AND INTEGRATIVE FUNCTIONS OF NERVOUS SYSTEMS
}

$\mathrm{D}^{\mathrm{s}}$ URING the Southampton meeting of the British Association, Section D (Zoology) discussed some recent progress in our knowledge of nervous systems, particularly the relationship between structure and function. The first two spoakers wero concerned with invertebrate animals, many of which continue to provide preparations and information which is of value in furthering our understanding of basic nervous mechanisms. The second two speakers presented data from two fields of study which have greatly advanced our knowledge of the mammalian brain during recent years.

Dr. E. A. Robson (Department of Zoology, Cambridge) began appropriately with a quotation from Sherrington's classic The Integrative Action of the Nervous System, in which he said "in the multicellular animal . . it is nervous action which par excellence integrates it, welds it together from its components, and constitutos it from a more collection of organs an animal individual. This integrative action in virtue of which the nervous system unifies from separate organs an animal possessing solidarity, an individual, is the problem before us . . . In discussing coelenterate nervo-nets, Dr. Robson pointed out that these animals are without a central nervous systom and yet show a remarkable degree of integration in their behaviour. Histological studies of the nervous systems of jelly fish and sea anemones by Schäfer and the Hertwigs in 1878 had shown the cxistence of neurones as separate units. Their work, in fact, providod oarly support for the neurono thoory, which was only established later from data on the vertebrate nervous system. Dr. Robson went on to review the classicul work of Pantin and his co-workers which, by means of clectrical stimulation and mechanical recording techniques, had made it possiblo to interpret the behaviour of coelenterates in torms of the properties of neuromuscular and interneural facilitation. Thus it had provided valuable physiological support for the view that the nerve net is synaptic. The distinction was made between through-conducting systems responsible for the protective response of a sea anemone and the swimming beat of a medusa, and tho more localized responses which are coordinated by a net in which the number of synapses to be crossed appears to be greater.

In addition to those reflex responses, coelenterato behaviour is also characterized by inherent activity, first studied in detail by Batham and Pantin using the sea anemone Metridium. This animal shows rhythmic expansion and shortening which are usually synehronized with dawn and dusk, but may persist in total darkness for at least three woeks. The interaction of this and other autonomous phases of activity with responses to environmental stimuli can produco impressive scquences of behaviour-well illustrated by the observations of Ross on the transference of Calliastis from one whelk shell to another.
More recent advances in our knowledge of the coelenterate nervous system have resulted from the application of electrical recording methods to these animals. This was first achieved by Horridge, who recorded a nerve impulse in the through-conducting system of the jellyfish, Aurelia. More recent work has shown that a variety of electrical phenomena can be recorded using micro-electrode techniques. With electrodes in the foot of Hydra, Passano and MeCullough recorded a constant background of potentials which arose from this region. They could be excited by light and were possibly endogenous to the endodermal nerve net. They also observed bursts of impulses which were associated with spontaneous longitudinal contrac. tions. These arose from tho hypostome and were initially inhibited by light and possibly occur in the endodermal nerve net. Other potentials were associated with attachment of the tentacles during locomotion and other tentacular movements. Clearly there is considerable electrical activity in the nervous system of Hydra and exactly what mechanisms are involved is only now being investigated. It suggests that the behaviour of Hydra is controlled by a hierarchy of pacemakers which are in turn influenced by sensory stimuli. Josephson has found a similar picturo in the marine hydroid Tubularia, where rhythmical potentials often not directly related to visible behaviour are also observed. The application of these methods has revcaled new physiological probloms, since, for example, it appears that there are sometimes more than two separate conducting systems, although only two separato nerve nots, ectodermal and endodermal, have been recognized structurally. Dr. Robson suggested that other structures such as musclo fields may have a role as conducting systems.

It seems probable that, as with other invertebrates, the ability to 'tune in' to the norvous activity will give us far more detailed information about the integrative mechanisms where previously our information was based largely on the overt behaviour and responses of the animal. Tho application of similar techniques has very recently led to the recording of the electrical signs of nervous action in the remaining major group of animals in which it had not been achieved - the Echinoderms, which also rely a great deal upon a nerve net.

Dr. G. M. Hughes (Department of Zoology, Cambridge) explained that tho title of his talk, "Electrophysiological Anatomy of Invertebrate Nervous Systems", had been chosen to emphasize the way electrophysiological techniques developed, for the investigation of detailed mechanisms of norvous function is of value in establishing both gross and micro-anatornical features of nervous organization. Thus, in the crayfish they have shown that the areas innervated by a given segmental nerve are not confined to a single segment. Furthermore, the scgmental pattern of the sensory innervation of the abdomen is out of phase with the segmentation of the ganglia supplied by the 
sensory nerves. Electrophysiological studies are of particular value not only in establishing the anatomy of the system but also because they give information about the functional connexions between neurones. In experiments on the abdominal cord of the dragonfly larva and crayfish, use has been made of the separation of synaptic regions in different ganglia by splitting the connectives between ganglia and so recording the effect of inputs from different segments. Several types of sensory-interneurone connexion have been established; in a common one a given interneurone makes connexions with homologous sensory inputs entering several ganglia. Impulses initiated in these separate synaptic regions will collide following simultaneous stimulation of inputs to different ganglia. Such interneurones produce different patterns of impulses depending on the particular way the sensory field is stimulated.

The sea hare, Aplysia, has provided many useful neurophysiological preparations, one of which has recently been found during electrophysiological studies of the anatomy of the two largest cells on each side of the nervous system. One of these cells (LGC) is in the left pleural ganglion and the other (RGC) on the right side of the abdominal ganglia. These two cells send axonal branches into many of the nerves which innervate the foot and parapodia.

Stimulation of any of these nerves is followed by the recording of an antidromic spike in the cell soma and, conversely, an impulse may be recorded in the axon when the soma is stimulated. It has recently been shown that both cells innervate not only ipsilateral regions but also send branches to the contralateral nerves. Direct stimula. tion of the RGC in whole animal preparations does not lead to any muscular contraction. They are probably homologous cells whose different positions in the CNS result from the processes of torsion and detorsion during development. Functionally, a direct synaptic connexion between the two cells has been established from the RGC to the LGC but not vice versa, and both cells have several common interneurones. Both giant cells show a remarkable degree of habituation in their response to synaptic inputs, there being a marked fall in the response between the first and second stimuli.

Other Aplysia neurones have shown that conduction between two axonal branches is not always equally possible in both directions. This provides a possible mechanism whereby only a part of the total output from a given neurone might be excited by a given input. In this way a single neurone could sub-serve the functions usually ascribed to several neurones. Some recent observations have also suggested that similar mechanisms may be involved in the separate function of portions of neurones in arthropod central nervous systems. Such devices, together with variations in synaptic properties, will be of great value as integrative mechanisms. They also suggest how animals with relatively small numbers of neurones may be able to accomplish more complicated feats of integration than might have been supposed had each neurone functioned in an all-or-none way and as a single unit according to the neurone doctrine.

In concluding the discussion of the first half of the symposium, the President of the Association (Lord Brain) emphasized how, despite many advances in the techniques being used for these investigations, the experimental methods were based on those established by the classical studies of Sherrington.

Dr. J. de C. Downer (Departments of Psychology and Anatomy, University College, London) prefaced his paper on "The Role of Brain Commissures in Interhemispheric Transfer of Training" with a point of view which is generally accepted by students of brain and behaviour, namely, that the ability of an animal to learn depends on the structure and organization of the brain. Hence an obvious technique is to remove specific regions of the brain and to test the ability of the animal to retain some previously learned solution and, if lost, its ability to re-learn. Results of such investigations have proved extremely difficult to interpret but have demonstrated quite clearly that the vertebrate brain, as with some invertebrate nervous systems, is characterized by the ability of one part to take over the function of another following its ablation. From some classical experiments, Lashley concluded that sub-cortical learning does not occur when the cortex is present and that it can only take place following cortical damage.

Dr. Downer went on to describe experiments in which he had made use of the split brain preparation introduced to this type of study by Sperry and Myers. Their experiments elearly showed that when the visual input is restricted to one-half of the brain (by cutting the crossed optic nerve fibres and occluding one eye) visual information normally crosses from the half receiving the input to the other half via the large commissures. This provided a function for the largest of these (corpus callosum) which had long been conjectural. In the primates, however, it does not connect the visual receiving areas across the midline and there is little evidence to suggest that information can pass from the visual association area to the primary visual receiving area. There seemed a distinct possibility that the visual association area might contain the residual memory store. Experiments designed to test this hypothesis led to the surprising results that, after ablation of this region, a monkey continued to be able to transfer a previously learned ability, and make a given visual discrimination even when the input was confined to the untrained and damaged side. However, such an animal was unable to transfer new discriminations learned through the intact side of the brain. Evidently the memory trace must lie outside the association area removed, but in its absence a new memory trace could not be established through the transfer training technique. However, by restricting the input to that eye which fed into the damaged side of the brain, it was found that the transfer of visual learning from the damaged to the intact side of the brain could still occur.

Using a second group of monkeys, an investigation was made of the role of other neocortical areas in the above experiments (optic chiasma sectioned). All the neo-cortex of the untrained half of the brain was removed except for the primary visual receiving area and motor cortex. When new visual discriminations had been learned on the intact side they did not transfer to the damaged side. The latter could still be trained but usually required about five times as many trials. After training the damaged side it was unexpectedly found that the learned response transferred to the intact side. These experiments showed, therefore, that visual learning does not transfer from the intact half of the brain to that from which most of the cortex outside the primary visual receiving area had been removed. However, if the side sustaining the ablations is trained, the learned response does transfer to the intact side.

A plausible explanation is that when the intact sido is being trained the neo-cortex is primarily involved in the learning process. Transfer to the damaged side cannot occur since the noocortical commissures have degenerated. But when the damaged side is trained, sub-cortical structures, probably the hippocampus, sub-serve the learning process and learning transfers to the opposide side via the hippocampal commissures which remained intact in these experiments.

The final talk in the symposium was given by Dr. E. G. Gray (Department of Anatomy, University College, London), who dealt with the relationship between structure and function of the nervous system at a sub-cellular level. He pointed out that during the past ten years electron microscopy has completely changed our understanding of the fine structure of the brain and the all-important transmission regions, the synapses. Before this time, light microscope preparations of silver-stained brain tissues had shown synapses as small bulbs or ring-shaped structures. But the electron microscope has shown that these struc- 
tures are fine bundles of protein neurofilaments within the axon and its presynaptic terminal expansion. The light microscope did not show their true shape or the various organelles present at the synapse itself. Most pre-synaptic terminals do not contain neurofilaments and are not observed in silver proparations. Consequently there was a considerablo undorestimation of the number of synapsos in a given region. In particular, the apparent absence of typical synapses in the cerebral cortex caused much embarrassmont to theorists on cortical function. Howevor, abundant synaptic contacts in the cortox have now been revealed by the eloctron microscope.

Electron microscopy has also revealed the presonce of numerous synaptic vesicles in the pre-synaptic terminals and it is believed by some that these contain chernical transmitters (acetylcholine at some synapses) which are reloased into the synaptic eleft by an impulse in the presynaptic fibre. Recent work has revoaled regularly shaped dense structuros at the synaptic membrane and these may function as focal points or pores for tho liberation of transmitter substances. Most vortebrate and invertebrate synapses show this chomical-release method of synaptic transmission, but certain specialized synapses depend on direct electrical transmission from pre- to post-synaptic elements without involving a chemical-release mochanism.
In some cases these synapses appoar to differ from chemically transmitting synapses in that there is a fusion of the pre- and post-synaptic membranes.

Much morphological variation of pro- and postsynaptic components can bo obsorved with the electron microscope. Espocially interesting is the occurrence of numerous spinous outgrowths, once regarded as artefacts in Golgi-method impregnation material, which can be seen to have synaptic contacts at their tip. A peculiar organelle, 'the spine apparatus', occurs within the spine cytoplasm of neurones of the cerebral cortex. Comparablo apparatus is not known from other non-mammalian nervous tissue and at present we have no information about its function. Future advances may make it possiblo to distinguish excitatory from inhibitory synapsos and perhaps to detect the sort of structural modifications which probably take place at synaptic membranes during the process of learning.

The whole symposium was concluded by a very profitable discussion. It had certainly given a broad view of the integrative action of the norvous system. Furthermore, as Sir Wilfrid Le Gros Clark pointed out in his final remarks, it had demonstrated the value of the integration of anatomy, zoology and physiology.

\title{
ACCURACY OF SEA GRAVITY SURVEYS: COMPARISONS OF SHIPBOARD AND SUBMARINE GRAVITY VALUES
}

\author{
By DR. B. D. LONCAREVIC
}

\author{
Bedford Institute of Oceanography, Dartmouch, Nova Scotia
}

\begin{abstract}
CHIPBOARD gravimeter surveys are the latest tool of $\checkmark$ marine geophysics ${ }^{1}$. The accuracy of any particular survey is very difficult to assess, and very often can only be estimated by intelligent guessing because of tho uncertainty of the navigational correction (Eötvös correction) ${ }^{2,3}$. When sufficiently close to land, the ship's position can be determined either by using direct fixes on shore marks or by using procision electronic positioning devices (Decca). An estimate of the accuracy of the shipborne gravimeter can be obtained in this case either by evaluating the gravity differences at track intersections ${ }^{4}$ or by comparing shipboard values with those obtained by means of gravimeters placed on the bottom of the sea $a^{5,0}$. The latter technique is the only one which provides a means of comparing shipboard values with reference values which are at least an order of magnitude moro accurate. However, tho bottom gravity measurements can be made only in the depth of water normally found on the continental shelves. Yet the largost part of oceanic survey is carried out over abyssal depths and a method is required for checking the sea gravimetor measurements obtained over the deep ocean.
\end{abstract}

If a buoy can be placed in deep water and a close survey is carried out within radar rango of it ${ }^{7}$, then the accuracy of the sea gravimoter may be evaluated by comparing the gravity values obtained on different tracks at their intersections ${ }^{8}$. This is a special case, and for most oceanic surveys the only available means of checking is to attempt to re-occupy previously established submarine pendulum stations. This article describes the checks during the first two years of operation of a Graf-Askania sea gravimeter by the Department of Geodesy and Geophysics, University of Cambridge.

The method of submarine gravity measurements ${ }^{9,10}$ has been establishod for more than thirty years and the root mean square error of a single station observation has been estimated as \pm 3.4 mgals (ref. 11) or as \pm 3.6 mgals (ref. 12). Tho main difficulty in using submarine stations as check points is due to the inherent problem of locating a ship at gea: our methods of celestial navigation have not changed for at least a hundred years. If the weather conditions are ideal and the drift due to currents and wind can be allowed for, then the ship's position can be estim. ated with an accuracy of 1-2 miles. Viewing conditions are seldom ideal even when the sky is not completely overcast, thereby increasing the error in the estimated position. In the past the location of submarine stations was usually chosen with particular geophysical problems in mind: to investigate the crustal structure over the continental slope, the seamounts, deep trenches or midocean ridges. In each case the horizontal gradients are large, and small error in the position of either the submarine or ship makes comparison between their respective gravity values moaningless. In spite of all the foregoing arguments, re-occupation of submarine stations gives $u$ worth-while check, and should be attempted whenever the research vessel's track can be conveniently arranged to pass near a known submarine pendulum station.

The eastern Atlantic is a particularly good area for checks of the shipborne gravimeter because of the large number of submarine stations which are fairly uniformly distributed. Forty-seven of these stations were re-occupicd during the 1960 cruise of R.R.S. Discovery $I I$ and the 1961-62 cruise of H.M.S. Owen ${ }^{13}$. Thirty-eight comparisons were available for evaluation and they are presented in Fig. 1 and Table 1.

The details of comparisons are given in Table 1 so that other investigators can make further checks if thoro is an opportunity to do so. The serial numbers in column 1 have beon arbitrarily assigned. The distances, given in column 5, between the reported position of the submarine station and the nearcst approach of the ship's track have a probable error of two miles due to navigational uncertaintics in position. The gravity values are the free air anomalies and tho throo values given for tho ship are $10 \mathrm{~min}(1.5-1.8$ nautical miles) readings around the 